\title{
EFFICIENT MODELS FOR WIND TURBINE EXTREME LOADS USING INVERSE RELIABILITY
}

\author{
K. Saranyasoontorn and L. Manuel \\ Department of Civil Engineering, University of Texas at Austin, \\ Austin, Texas 78712, USA
}

\begin{abstract}
The reliability of wind turbines against extreme loads is the focus of this study. A procedure to establish nominal loads for use in a conventional load-and-resistance-factor-design (LRFD) format is presented. The procedure, based on an inverse reliability approach, permits inclusion of randomness in the gross wind environment as well as in the extreme response given wind conditions. A detailed example is presented where three alternative nominal load definitions are used to estimate extreme bending loads for a $600 \mathrm{~kW}$ three-bladed horizontal-axis wind turbine. Only operating loads - here, flapwise (out-of-plane) bending moments - at a blade root are considered but the procedure described may be applied to estimate other loads and response measures of interest in wind turbine design. Results suggest that a full random characterization of both wind conditions and short-term maximum response (given wind conditions) will yield extreme design loads that might be approximated reasonably well by simpler models that include only the randomness in the wind environment but that account for response variability by employing appropriately derived "higher-than-median" fractiles of the extreme bending load conditional on inflow parameter values.
\end{abstract}

\section{INTRODUCTION}

In reliability-based design, the conventional load-and-resistance-factor-design (LRFD) procedure involves the scaling of a nominal load, $L_{n o m}$, by a load factor, $\gamma_{L}$, and the scaling of a nominal (or characteristic) resistance, $R_{n o m}$, by a separate resistance factor, $\phi_{R}$, to account for possible overload and understrength. This is done in the following checking equation which aims to provide for design against a specified limit state:

$$
\phi_{R} R_{\text {nom }} \geq \gamma_{L} L_{\text {nom }}
$$

Calibration of both the scaling factors and the associated definitions of $L_{n o m}$ (for example, as a load level associated with a specified return period) and $R_{\text {nom }}$ are needed to guarantee specified probabilities of meeting the limit state under consideration. For wind turbine generator systems, a similar reliability-based design format is employed in the IEC 61400-1 code where the load factor, $\gamma_{L}$, and the resistance factor, $\phi_{R}$, in Eq. (1) are combined into a single factor, $\gamma_{n}$, that represents a safety factor for "consequences of failure" (IEC/TC88 614001 ed. 2, 1998).

Various alternative definitions of the nominal load level may be employed to account for load variability. In the present study for wind turbine extreme loads, we consider three such load definitions related to assumptions used in representing load uncertainty in Eq. (1). The key uncertainties in the design of wind turbines arise from (i) the gross inflow parameters, usually taken to be the ten-minute mean horizontal wind speed at hub height and the standard deviation (also a measure of turbulence) of the same wind speed process; and (ii) the ten-minute maximum load/response conditional on the inflow parameters.

In the following, we refer to the inflow parameters as simply mean and standard deviation of wind speed, with 
an understanding that we are referring to statistics of the time-varying horizontal wind speed process at hub height. We consider an extreme flapwise (out-of-plane) bending moment at the blade root as the load of interest. Our objective is to establish appropriate nominal design loads for several target reliability levels (or, equivalently, for several different return periods). These loads defined with different degrees of complexity in the assumptions on the variability of the key random variables are compared and insights gained from this comparison are discussed to aid in development of a rational procedure for establishing design loads.

\section{STRUCTURAL RELIABILITY AND INVERSE METHODS}

The First-Order Reliability Method (FORM) is essentially an "analysis" approach whereby one seeks a "solution" which corresponds to a most-likely-to-fail combination of the various load and resistance quantities (for details related to FORM, see Madsen et al, 1986). The probability of failure associated with this combination is easily estimated but it is not known a priori. An iterative approach in such "forward-FORM" analyses where one adjusts, for example, the resistance/capacity level to yield a specified reliability or probability of failure would constitute a "design" procedure. This iterative procedure, however, is tedious in many situations.

An alternative approach is to use "inverse reliability" techniques that avoid multiple forward-FORM iterations. Many recent studies have proposed procedures for these techniques and/or have applied them to different design problems. Winterstein et al (1993) developed an Inverse First-Order Reliability Method (Inverse-FORM) procedure that is based on the use of "environmental contours" that uncouple environmental random variables from structural response. Using examples related to offshore structures, they showed how this method may be applied to estimate design loads associated with specified target reliability levels. Der Kiureghian et al (1994) proposed an extension of the Hasofer-Lind-Rackwitz-Fiessler FORM algorithm that uses a search direction and a merit function as part of a very efficient procedure that finds the design point associated with a target reliability. Li and Foschi (1998) showed, using several examples, how multiple design variables may be treated in an inverse reliability procedure.

Recently, Fitzwater et al (2003) applied inverse reliability methods for extreme loads on pitch- and stallregulated wind turbines where they employed results from aeroelastic simulations to represent the response given inflow conditions. The response variable there was treated as deterministic allowing the use of 2-D environmental contours based on Winterstein et al (1993). In the present study, our interest is in estimating design extreme flapwise bending loads for a $600 \mathrm{~kW}$ three-bladed horizontal-axis wind turbine that was previously studied by Ronold and Larsen (2000), where results from field measurements were reported and probabilistic models for response (loads) conditional on inflow conditions were presented. The distinction between the present study and that by Fitzwater et al (2003) is that we propose alternative nominal load definitions (one of which includes response variability) and we employ field data instead of simulations in developing parametric models for the random response conditional on inflow. Our alternative load definitions are based on what will be described as 1-D, 2-D, and 3-D models which refer to how the inflow and response variables are treated - i.e., whether deterministic or random. The full 3-D characterization of variables refers to modeling of all variables as random, while the other two models refer to simplifications where, in the 1-D case, only mean wind speed is modeled as random and, in the 2-D case, the mean and standard deviation of the wind speed are both modeled as random (but response is not). A discussion of enhancements to the 2-D model that reflect omitted randomness in the response (conditional on inflow) is presented that is based on the use of omission sensitivity factors (Madsen, 1988; Winterstein et al, 1993). Such enhancements approximately get at the full randomness of the response by employing "higher-than-median" fractiles of the extreme bending load conditional on inflow - these are improvements on the ordinary 2-D model that uses a deterministic representation of response or, effectively, median levels of load conditional on inflow.

The alternative nominal load definitions are presented next, along with a general background on the InverseFORM framework for establishing design loads. Design load levels for different return periods based on the alternative models are compared for the $600 \mathrm{~kW}$ wind turbine described by Ronold and Larsen (2000). 


\section{ALTERNATIVE MODELS}

Our interest here is in obtaining estimates of a nominal load, $L_{n o m}$, for failure in an extreme/ultimate limit state associated with bending of a wind turbine blade in its flapwise (out-of-plane) mode. We assume that the uncertainty in these extreme bending loads depends on inflow parameters and on short-term maximum loads conditional on the inflow parameters. As stated previously, the inflow parameters that characterize the wind are the ten-minute mean wind speed at hub height, $U_{10}$, and the standard deviation, $\sigma$, of the wind speed. The load, $L_{n o m}$, considered here is the extreme flapwise bending moment at the root of a turbine blade corresponding to a specified return period of $T$ years. From the field data on the wind turbine considered, ten-minute extremes of the random flapwise bending moment, $B M_{\text {ext }}$, are used to derive the nominal load, $L_{n o m}$. For convenience, in discussions that follow, we will refer to the three short-term (i.e., ten-minute) random variables, $U_{10}, \sigma$, and $B M_{e x t}$ as $X_{1}, X_{2}$, and $X_{3}$ that make up the physical random variable space, $\boldsymbol{X}$.

Consider a situation where the joint probability description of $X_{1}, X_{2}$, and $X_{3}$ is available in the form of a marginal distribution for $X_{1}$ and conditional distributions for $X_{2}$ given $X_{1}$, and for $X_{3}$ given $X_{1}$ and $X_{2}$. The simplest definition of $L_{\text {nom }}$ is based on a representative load derived from the $T$-year value of the random $X_{1}$ (mean wind speed) alone and consideration of $X_{2}$ (standard deviation on wind speed) and $X_{3}$ (ten-minute extreme bending load) only by representing these as conditional median values. In this model for $L_{n o m}$, uncertainty is neglected in both $X_{2}$ and $X_{3}$. A second definition might be based on a representative $T$-year load that includes randomness in both $X_{1}$ and $X_{2}$ but still neglects uncertainty in the short-term load, $X_{3}$. Again, this load is held fixed at its median level given $X_{1}$ and $X_{2}$. Finally, a definition for nominal load could be based on the "true" $T$ year nominal load including uncertainty in all of the three variables. We refer to these definitions as "1-D", "2D", and "3-D" probabilistic models respectively. The nominal loads based on these models can be expressed as:

$$
L_{n o m, 1 D}=\hat{X}_{3}\left(X_{1}, \hat{X}_{2} \mid X_{1}\right) ; \quad L_{n o m, 2 D}=\hat{X}_{3}\left(X_{1}, X_{2} \mid X_{1}\right) ; \quad L_{n o m, 3 D}=X_{3}\left(X_{1}, X_{2} \mid X_{1}\right)
$$

in which $\hat{X}_{2}$ represents the median of $X_{2}, \hat{X}_{3}$ denotes the median of $X_{3}$, and "|" denotes a conditional sign (for

example, $\hat{X}_{2} \mid X_{1}$ refers to the median value of $X_{2}$ given $X_{1}$ ). The load factor, $\gamma_{L}$, in equation (1) will be appropriately different for each of the nominal load definitions above in order to ensure that the design checking equation leads to consistent reliability estimates in each case. In the following, we use an example wind turbine and available field data, and then derive and compare nominal wind turbine bending loads based on these three probabilistic models.

It is possible to establish nominal loads by direct integration involving the conditional short-term maximum load distribution (given inflow conditions) and the joint density function of the inflow variables. For the right choice of $L_{n o m}$, integration will lead to the desired target probability of failure, $P_{f}$, as follows:

$$
P_{f}=P\left[X_{3}>L_{n o m}\right]=\iint_{X_{1}, X_{2}} P\left[X_{3}>L_{n o m} \mid X_{1}, X_{2}\right] f_{X_{1}, X_{2}}\left(x_{1}, x_{2}\right) d x_{1} d x_{2}
$$

where $f_{X_{1}, X_{2}}\left(x_{1}, x_{2}\right)$ is the joint probability density function of $X_{1}$ and $X_{2}$. Using Eq. (3) to obtain the nominal load would provide the "exact" load but would be computationally expensive in practical situations. Also, not much would be learned about the inflow conditions that bring about this load. Inverse reliability procedures, on the other hand, are approximate but less computationally intensive and have an important advantage in that they offer useful insights into the derived load and about the associated inflow conditions. In particular, we will use the Inverse-FORM approach proposed by Winterstein et al. (1993). An overview is presented next of how this method based on environmental contours works in the current study.

Consider a sphere of radius equal to the target reliability index, $\beta$, in an $n$-dimensional space describing independent standard normal variables, one for each of the physical random variables in the problem of interest. If at any point on this sphere, a tangent hyperplane were drawn, the probability of occurrence of points on the side of this hyperplane away from the origin is $\Phi(-\beta)$, where $\Phi($ ) refers to the Gaussian cumulative distribution function. Since each point on the sphere is associated with the same reliability level, if the nominal load desired is also for this same level, the points on the sphere can be systematically searched until the largest nominal load is obtained. Transformation from the standard normal $(\boldsymbol{U})$ space to the physical random variable $(\boldsymbol{X})$ space is necessary in order to obtain the nominal load. This is achieved by using the Rosenblatt transformation (Rosenblatt, 1952). In the present study, a complete probabilistic representation of the random variables requires that $n$ is equal to 3. However, we shall see how only the 3-D model in Eq. (2) retains all three random 
variables. The median value indicated in the definition of $L_{n o m, 2 D}$ in Eq. (2) is equivalent to forcing the variable $U_{3}$ to be zero; and for $L_{n o m, 1 D}$, it is equivalent to forcing the two variables, $U_{2}$ and $U_{3}$ to both be zero. This has the effect of causing 2-D and 1-D models for $L_{n o m}$ to be obtained by reducing the dimension of the random variable space and the 3-D sphere to a circle and a point, respectively.

In summary, the 1-D model assumes that the ten-minute mean wind speed, $X_{1}$, is random but neglects the variability in the standard deviation of the wind speed, $X_{2}$, and in the ten-minute maximum response, $X_{3}$. Thus, in $\boldsymbol{U}$ space, the $n$-dimensional "sphere" is a degenerate single point, $u_{1}=\beta, u_{2}=u_{3}=0$. Similarly, the 2-D model assumes that only $X_{1}$ and $X_{2}$ are random; in $\boldsymbol{U}$ space, the $n$-dimensional "sphere" is a degenerate circle, $u_{1}{ }^{2}+u_{2}{ }^{2}$ $=\beta^{2} ; u_{3}=0$. The 3 -D model treats all three variables as random and is represented by the 3-D sphere, $u_{1}{ }^{2}+u_{2}{ }^{2}+$ $u_{3}{ }^{2}=\beta^{2}$. Geometric representations for the three models are shown in Fig. 1. Because our target reliability is specified in terms of a return period (equal to $T$ years) associated with the nominal load, $L_{n o m}$, note that since $X_{3}$ is defined as the extreme value in ten minutes, we need to determine the appropriate value of $\beta$ to be used in the Inverse-FORM approach described by the $1-\mathrm{D}, 2-\mathrm{D}$, and 3-D models. This is done by setting $\beta=\Phi^{-1}\left(1-P_{f}\right)$ where $\beta$ and $P_{f}$ are related to the target return period ( $T$ years) and the number of ten-minute segments in $T$ years. Assuming independence between extremes in the various ten-minute segments, for the three return periods studied here corresponding to 1,20 , and 50 years, the values of $P_{f}$ are $1.90 \times 10^{-5}, 9.51 \times 10^{-7}$, and $3.81 \times 10^{-}$ ${ }^{7}$, respectively, and the values of $\beta$ are $4.12,4.76$, and 4.95 , respectively.

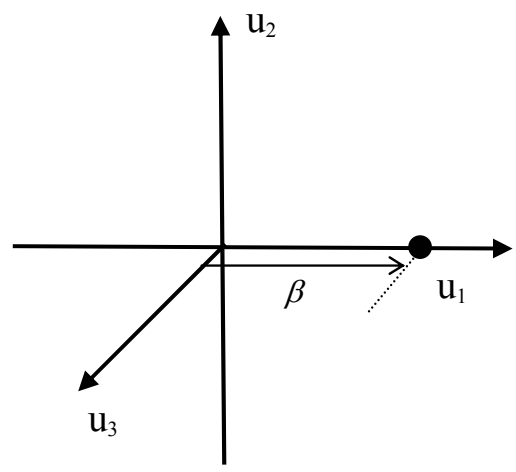

(a)

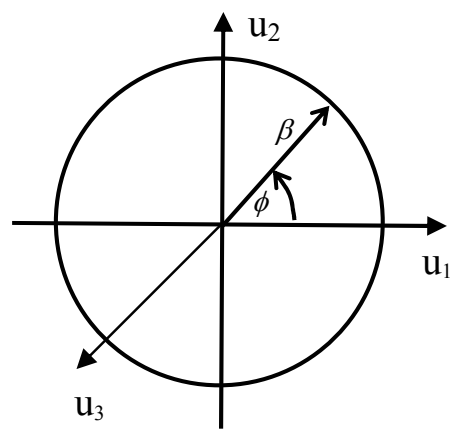

(b)

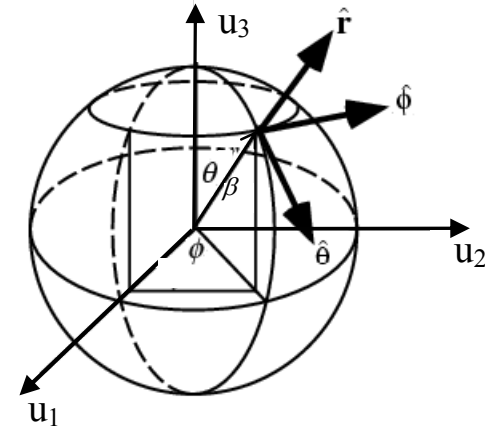

(c)

Figure 1: Geometric representation of the $n$-dimensional "sphere" in $\boldsymbol{U}$ space associated with a prescribed reliability index, $\beta$, for three cases: (a) 1-D model $(n=1)$; (b) 2-D model $(n=2)$; and (c) 3-D model $(n=3)$.

\section{NUMERICAL STUDIES}

The wind turbine considered in this study is a $600 \mathrm{~kW}$ stall-regulated horizontal-axis turbine with three $21.5 \mathrm{~m}$ long rotor blades and a hub height of $44 \mathrm{~m}$. This turbine has been the subject of previous studies and is one for which field data as well as extrapolated design loads have been derived by Ronold and Larsen (2000). The probabilistic models for the short-term maximum flapwise bending load and the environmental variables are used here with slight changes in some parameters.

The distribution of the extreme flapwise bending moment is obtained on the assumption that the underlying bending moment process may be characterized by its four statistical moments (i.e., mean, $\mu$; standard deviation, $\sigma$, skewness, $\alpha_{3}$; and kurtosis, $\alpha_{4}$ ) and that this non-Gaussian process (and its extremes) may be mapped to a Gaussian process (and its extremes) using a Hermite moment transformation. Based on field data, the first two statistical moments and the zero-crossing rate of the flapwise load were represented as functions of $X_{1}$ and $X_{2}$ as shown in Fig. 2. Other information related to the distributions for $X_{3} \mid X_{1}, X_{2}$ as well as for $X_{1}$ and $X_{2} \mid X_{1}$ is summarized in Table 1.

\section{1-D Model}

Here, only the ten-minute mean wind speed, $X_{1}$, is modeled as random, while the standard deviation of wind speed, $X_{2}$, and the ten-minute maximum response, $X_{3}$, are held at their (conditional) median levels. Knowing the reliability index, $\beta$, associated with a prescribed return period and failure probability, one can immediately obtain the "point" corresponding to that failure probability in the standard normal $(\boldsymbol{U})$ space (see Fig. 1a) as follows: 


$$
u_{1}=\beta, u_{2}=0, \text { and } u_{3}=0
$$

The design point related to the nominal bending load in physical random variable space $(\boldsymbol{X})$ may be obtained using the Rosenblatt transformation:

$$
x_{1}=F_{X_{1}}^{-1}[\Phi(\beta)], x_{2}=F_{X_{2} \mid X_{1}}^{-1}[\Phi(0)], \text { and } x_{3}=F_{X_{3} \mid X_{1}, X_{2}}^{-1}[\Phi(0)]
$$

From Eq. (5), it is clear that median values of $X_{2}$ and $X_{3}$ lead to the nominal loads. This is illustrated graphically in Fig. 3, where, for example, the 20-year design load is seen to be $420.8 \mathrm{kN}-\mathrm{m}$ which is derived using a mean wind speed of $24.5 \mathrm{~m} / \mathrm{s}$ and a standard deviation of wind speed equal to $3.13 \mathrm{~m} / \mathrm{s}$. Similar results for the other return periods are also shown. Detailed results for all three return periods are summarized in Table 2 .

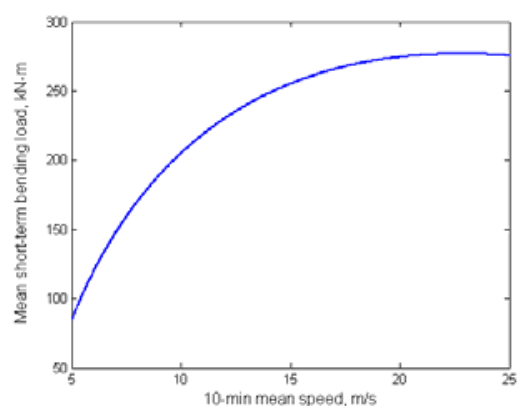

(a)

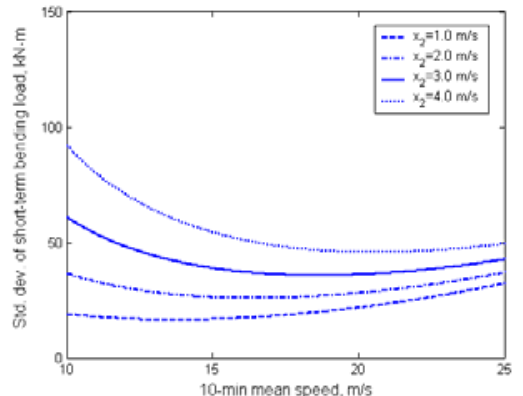

(b)

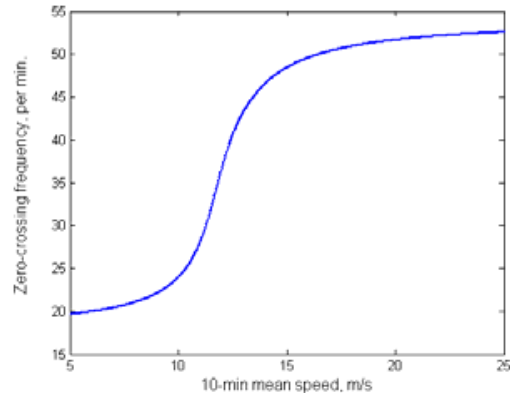

(c)

Figure 2: Dependence on mean wind speed $\left(X_{1}\right)$ of short-term maximum bending load model parameters: (a) mean value, $\mu$; (b) standard deviation, $\sigma$, and (c) zero-crossing frequency, $v$.

TABLE 1

DISTRIBUTIONS AND PARAMETERS FOR THE RANDOM VARIABLES.

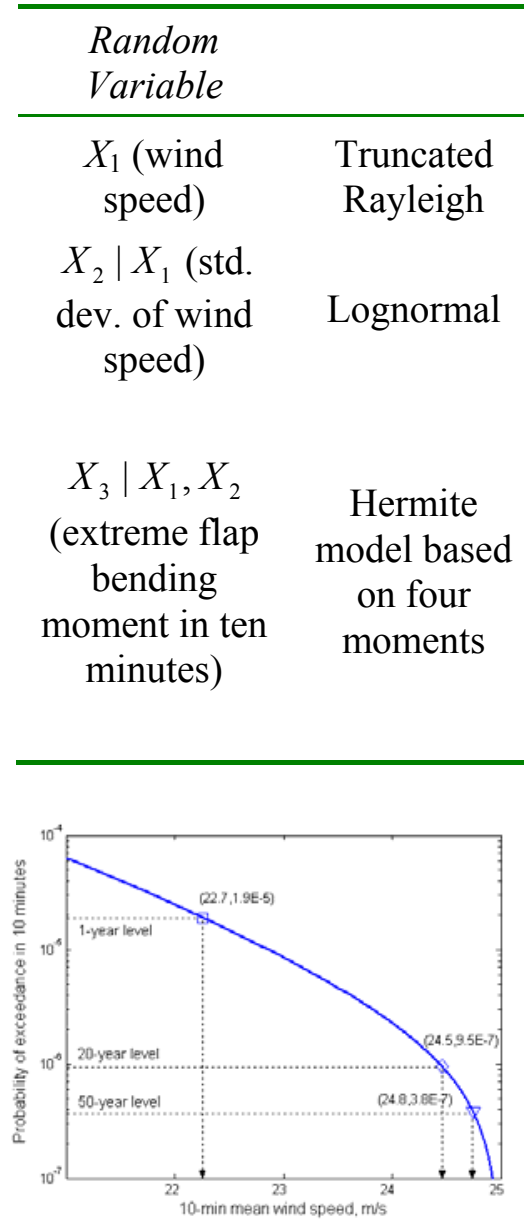

(a)
Distribution

$$
F_{X_{1}}\left(x_{1}\right)=\frac{1-\exp \left[-\left(x_{1} / A\right)^{2}\right]}{1-\exp \left[-\left(u_{c} / A\right)^{2}\right]}
$$$$
F_{X_{2} \mid X_{1}}\left(x_{2}\right)=\Phi\left(\frac{\ln x_{2}-b_{0}}{b_{1}}\right)
$$$$
F_{Y_{3}}\left(y_{3}\right)=\exp \left[-v T \exp \left(-\frac{y_{3}{ }^{2}}{2}\right)\right]
$$$$
x_{3}=x_{3}\left(y_{3}, \mu, \sigma, \alpha_{3}, \alpha_{4}\right)
$$

based on a transformation that relates the Gaussian extreme, $y_{3}$, to the non-Gaussian extreme, $x_{3}$.
Parameters (from Ronold and Larsen, 2000)

$$
\begin{gathered}
A=6.77 \mathrm{~m} / \mathrm{s}, u_{c}=25 \mathrm{~m} / \mathrm{s} \\
b_{0}=-2.1601+1.0326 \ln x_{1} \\
b_{1}=.0579+.6169 \exp \left(-.1709 x_{1}\right)
\end{gathered}
$$$$
\mu=\mu\left(x_{1}\right), \sigma=\sigma\left(x_{1}, x_{2}\right) \text {, and }
$$$$
v=v\left(x_{1}\right) \text { are shown in Fig. } 2 .
$$

$$
\begin{gathered}
\alpha_{3}=-0.0066 \\
\alpha_{4}=2.8174
\end{gathered}
$$

Figure 3: Procedure to estimate extreme loads based on the 1-D model: (a) evaluating $X_{1}$ for a specified return period; (b) evaluating $\hat{X}_{2}$ given $X_{1}$; and (c) evaluating $\hat{X}_{3}$ given $X_{1}$ and $\hat{X}_{2}$. 
TABLE 2

RESULTS SUMMARIZING DESIGN POINTS FROM THE 1-D, 2-D, AND 3-D MODELS FOR THREE RETURN PERIODS.

\begin{tabular}{|c|c|c|c|c|c|c|c|c|c|}
\hline \multirow{2}{*}{$\begin{array}{c}\text { Return } \\
\text { Period } \\
\text { (yrs) }\end{array}$} & \multicolumn{3}{|c|}{$1-\mathrm{D}$} & \multicolumn{3}{|c|}{$2-D$} & \multicolumn{3}{|c|}{$3-\mathrm{D}$} \\
\hline & $\begin{array}{c}X_{1} \\
(\mathrm{~m} / \mathrm{s})\end{array}$ & $\begin{array}{c}X_{2} \\
(\mathrm{~m} / \mathrm{s})\end{array}$ & $\begin{array}{c}X_{3} \\
(\mathrm{kN}-\mathrm{m})\end{array}$ & $\begin{array}{c}X_{1} \\
(\mathrm{~m} / \mathrm{s})\end{array}$ & $\begin{array}{c}X_{2} \\
(\mathrm{~m} / \mathrm{s})\end{array}$ & $\begin{array}{c}X_{3} \\
(\mathrm{kN}-\mathrm{m})\end{array}$ & $\begin{array}{c}X_{1} \\
(\mathrm{~m} / \mathrm{s})\end{array}$ & $\begin{array}{c}X_{2} \\
(\mathrm{~m} / \mathrm{s})\end{array}$ & $\begin{array}{c}X_{3} \\
(\mathrm{kN}-\mathrm{m})\end{array}$ \\
\hline 1 & 22.7 & 2.84 & 402.6 & 22.0 & 2.95 & 404.2 & 21.3 & 2.83 & 410.5 \\
\hline 20 & 24.5 & 3.13 & 420.8 & 24.1 & 3.33 & 423.8 & 23.1 & 3.12 & 436.8 \\
\hline 50 & 24.8 & 3.17 & 423.3 & 24.4 & 3.44 & 427.7 & 23.5 & 3.17 & 444.0 \\
\hline
\end{tabular}

\section{2-D Model}

Randomness in both of the environmental variables, $X_{1}$ and $X_{2}$, is now modeled while the load variable, $X_{3}$, is still assumed deterministic at its (conditional) median level. For a known reliability index, $\beta$, one can construct a "circle" in standard normal $(\boldsymbol{U})$ space (see Fig. 1b) as follows:

$$
u_{1}=\beta \cos \phi, u_{2}=\beta \sin \phi, \text { and } u_{3}=0 \text {; for }-\pi \leq \phi \leq \pi
$$

Since this circle lies in the plane, $u_{3}=0$, it is associated only with the environmental random variables and hence it is termed an "environmental contour." To obtain the design point, one needs to search the entire circle (by considering all values of the angle $\phi$ between $-\pi$ and $\pi$ ) so as to find the largest median response value for $X_{3}$. Applying the Rosenblatt transformation yields the design point in $\boldsymbol{X}$ space:

$$
x_{1}=F_{X_{1}}^{-1}[\Phi(\beta \cos \phi)], x_{2}=F_{X_{2} \mid X_{1}}^{-1}[\Phi(\beta \sin \phi)] \text {, and } x_{3}=F_{X_{3} \mid X_{1}, X_{2}}^{-1}[\Phi(0)]
$$

Every point on the environmental contour is such that the probability on the side of a tangent hyperplane at that point (away from the origin) is the same. However, each point is associated with a different "median" response. As shown in Fig. 4, for a 20-year return period, Eq. (7) maps the contour in $\boldsymbol{U}$ space to different median levels of response, whose maximum value is $423.8 \mathrm{kN}-\mathrm{m}$ which is associated with a mean wind speed of $24.1 \mathrm{~m} / \mathrm{s}$ and a standard deviation of wind speed equal to $3.33 \mathrm{~m} / \mathrm{s}$. The nominal bending load for this 2-D model can also be obtained by plotting separate iso-response curves of median values of $X_{3}$ together with the 2-D environmental contour in $\boldsymbol{X}$ space and locating the iso-response curve of highest value that intersects the 2-D environmental contour. It should be noted that the environmental contours and the iso-response curves may be constructed independently; hence, these contours are not turbine-specific. The turbine response/load is uncoupled from the environment. Environmental contours as well as iso-response curves are plotted in Fig. 5 from which it may be seen that the nominal loads are 404.2, 423.8, and $427.7 \mathrm{kN}-\mathrm{m}$, respectively, for 1-, 20-, and 50-year return periods. Detailed results for all three return periods are summarized in Table 2.

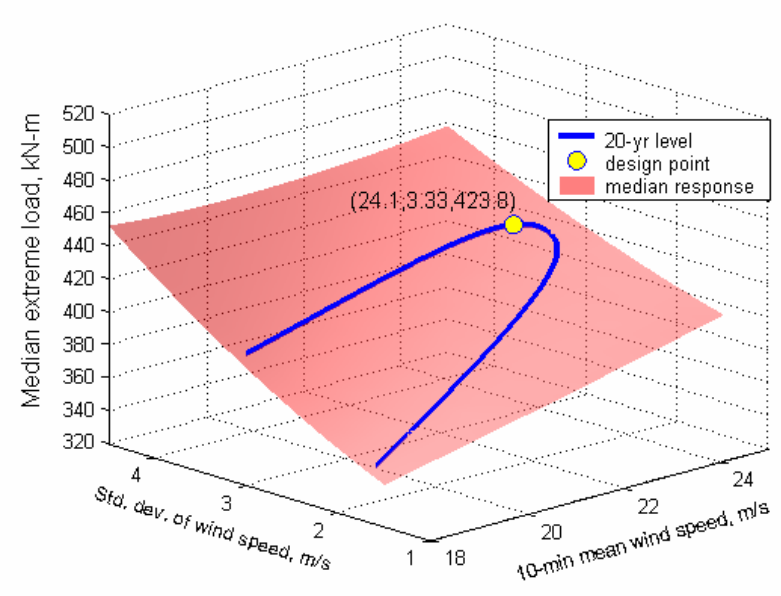

Figure 4: Median response surface, 20-year environmental contour, and design point.

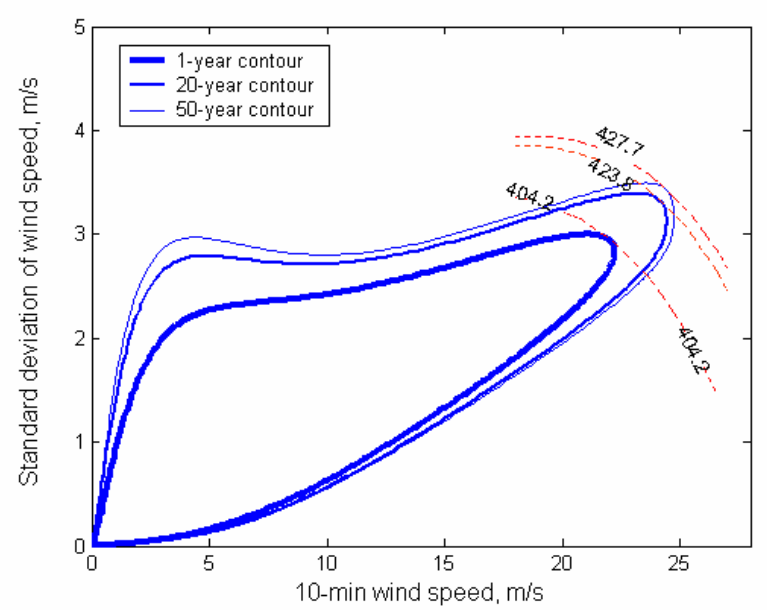

Figure 5: Environmental contours for different return periods and iso-response curves.

\section{3-D Model}

Randomness in all three random variables, $X_{1}, X_{2}$, and $X_{3}$ is now modeled. For a known reliability index, $\beta$, one can construct a sphere in standard normal $(\boldsymbol{U})$ space (see Fig. 1c) as follows:

$$
u_{1}=\beta \cos \phi \sin \theta, u_{2}=\beta \sin \phi \sin \theta \text {, and } u_{3}=\beta \cos \theta \text {; for }-\pi \leq \phi \leq \pi, 0 \leq \theta \leq \pi
$$


To obtain the design point, one needs to search the entire sphere (by considering all values of the angle $\phi$ and $\theta$ ) so as to find the largest response value for $X_{3}$. Applying the Rosenblatt transformation yields the design point in $\boldsymbol{X}$ space:

$$
x_{1}=F_{X_{1}}^{-1}[\Phi(\beta \cos \phi)], x_{2}=F_{X_{2} \mid X_{1}}^{-1}[\Phi(\beta \sin \phi)], \text { and } x_{3}=F_{X_{3} \mid X_{1}, X_{2}}^{-1}[\Phi(\beta \cos \theta)]
$$

As shown in Fig. 6, for a 20-year return period, Eq. (9) maps the sphere in $\boldsymbol{U}$ space to different levels of response, $X_{3}$ - the maximum value of response is found to be $436.8 \mathrm{kN}-\mathrm{m}$; for the 1-year return period, this maximum response is $410.5 \mathrm{kN}-\mathrm{m}$. Detailed results for all three return periods are summarized in Table 2 .

\section{Discussions and a Proposed Modified 2-D Model}

The three models presented above lead to different nominal loads as is seen in Table 2. In the table, it is seen that as would be expected, the nominal load levels increase with return period. For any return period, it is seen that the 1-D and 2-D models yield very slightly different loads; this is because the variable, $X_{2}$, the standard deviation of wind speed, is relatively unimportant compared to the mean wind speed, $X_{1}$. For the 20-year return period, the difference in nominal loads is only about $0.7 \%$. Greater differences are seen when the 3-D model is considered where short-term response uncertainty is included. The 3-D model 20-year loads are about 3\% higher than the 2-D loads. While for this particular problem, the difference between the 3-D model and the simpler models is small, this may not be the case when response variability (conditional on inflow) is large.

The 2-D model is of special interest because it uncouples the environment from the response. This is especially convenient when considering the same turbine in different environment conditions as well as when considering alternative turbines for a specified environment. Moreover, a modified 2-D model that employs omission factors (Madsen, 1988) to derive higher-than-median fractiles of $U_{3}$ (and hence $X_{3}$ ) can, with few additional calculations that follow a 2-D model analysis, reduce the error relative to the 3-D model. Results from such a 2$\mathrm{D}$ modified model are summarized in Table 3 where the FORM direction cosine, $\alpha_{3}$, which is related to the importance of the response variable was obtained by only computing a local gradient of the limit state function in the direction of $U_{3}$ (at the 2-D model design point). Figure 7 shows nominal load levels from all four models where it is clear that there is now negligible difference between the loads derived from the Modified 2-D model and those from the 3-D model.

TABLE 3

MODIFIED 2-D MODEL RESULTS FOR THREE RETURN PERIODS.

\begin{tabular}{|c|c|c|c|}
\hline $\begin{array}{c}\text { Return Period } \\
(\mathrm{yrs})\end{array}$ & $\begin{array}{c}\text { Direction cosine, } \\
\alpha_{3}\end{array}$ & $\begin{array}{c}\text { Modified Fractile for } \\
U_{3}\end{array}$ & $X_{3}(\mathrm{kN}-\mathrm{m})$ \\
\hline 1 & 0.26 & 0.71 & 409.9 \\
\hline 20 & 0.45 & 0.87 & 437.7 \\
\hline 50 & 0.51 & 0.91 & 445.5 \\
\hline
\end{tabular}

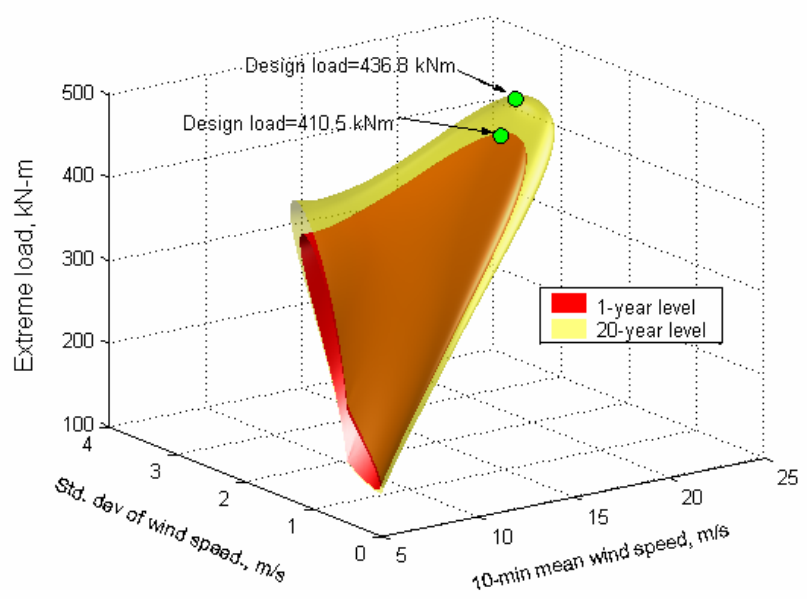

Figure 6: 3-D surfaces employed for deriving 1- and 20-year nominal loads with the 3-D model.

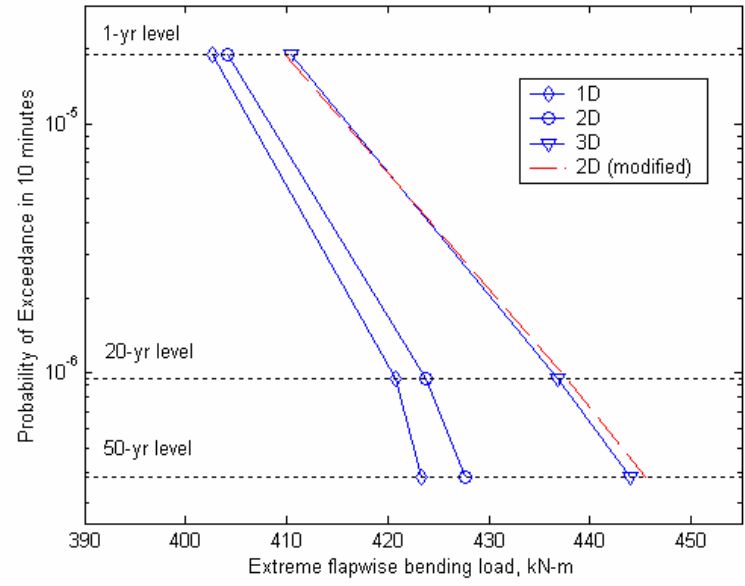

Figure 7: Extreme flapwise bending moment for 1-, 20-, and 50-year return periods based on a 1-D, 2-D, 3-D, and a modified 2-D model. 


\section{CONCLUSIONS}

We have presented a procedure to establish nominal loads for the design of wind turbines against ultimate limit states. Three alternative load models (termed 1-D, 2-D, and 3-D models) were compared. An inverse reliability approach was employed to estimate nominal design loads. For the $600 \mathrm{~kW}$ wind turbine considered, the environmental and response probabilistic descriptions were obtained from a study by Ronold and Larsen (2000). Extreme flapwise bending loads were studied and, for this turbine, it was found that the difference between the nominal loads derived from 1-D and 2-D models was very small since the standard deviation of wind speed at the hub height had a very small effect on the extreme bending load compared with the mean wind speed. Including uncertainty in the short-term maximum bending load conditional on inflow (in the 3-D model) caused somewhat higher loads than in the 1-D and 2-D models. The modified 2-D model that uses higher-than-median fractiles (derived by some additional calculations following the 2-D analysis) for the short-term response conditional on inflow was able to yield almost similar nominal loads as were obtained with the 3-D model.

The results presented were for a specific wind turbine, and conclusions drawn from this study are by no means generally applicable to other wind turbines or for other environmental conditions. However, the procedures outlined may be employed in any situation where the objective is to establish nominal loads for the reliabilitybased design of wind turbine components.

\section{ACKNOWLEDGEMENTS}

The authors gratefully acknowledge the financial support provided by Grant No. 003658-0272-2001 awarded through the Advanced Research Program of the Texas Higher Education Coordinating Board. They also acknowledge additional support from Sandia National Laboratories by way of Grant No. 30914.

\section{REFERENCES}

1. Der Kiureghian, A., Zhang, Y., and Li, C.-C. (1994). Inverse Reliability Problem, Journal of Engineering Mechanics, ASCE, Vol. 120, No. 5, pp. 1154-1159.

2. Fitzwater, L. M., Cornell, C. A., and Veers, P. S. (2003). Using Environmental Contours to Predict Extreme Events on Wind Turbines, Wind Energy Symposium, AIAA/ASME, pp. 244-258.

3. IEC/TC88 61400-1 ed. 2 (1998). Wind turbine generator systems-part 1: Safety Requirements, International Electrotechnical Commission (IEC).

4. Li, H. and Foschi, R. O. (1998). An Inverse Reliability Method and its Application, Structural Safety, Vol. 20, pp. 257-270.

5. Madsen, H. O. (1988). Omission Sensitivity Factors, Structural Safety, Vol. 5, pp. 35-45.

6. Madsen, H. O., Krenk, S. and Lind, N. C. (1986). Methods of Structural Safety, Prentice-Hall Inc., Englewood Cliffs, New Jersey.

7. Ronold, K.O. and Larsen G.C. (2000). Reliability-based Design of Wind-turbine Rotor Blades against Failure in Ultimate Loading, Engineering Structures, Vol. 22, pp. 565-574.

8. Rosenblatt, M. (1952). Remarks on a Multivariate Transformation, Ann. Math. Stat., Vol. 23, pp. 470-472.

9. Winterstein, S. R., Ude, T. C., Cornell, C. A., Bjerager, P. and Haver, S. (1993). Environmental Contours for Extreme Response: Inverse FORM with Omission Factors, Proc. ICOSSAR-93, Innsbruck. 